\title{
Integrins as a Critical Target in Determining Metastasis and Growth of Brain Tumor
}

\author{
Türker Çavuşoğlu ${ }^{1,2}$, Yiğit Uyanıkgil ${ }^{1,2}$, Kubilay Doğan Kılıç ${ }^{1}$ and Mehmet Turgut ${ }^{3^{*}}$ \\ ${ }^{1}$ Department of Histology and Embryology, Ege University, School of Medicine, Izmir, Turkey \\ ${ }^{2}$ Cord Blood, Cell-Tissue Research and Application Center, Ege University, Izmir, Turkey \\ ${ }^{3}$ Department of Neurosurgery, Adnan Menderes University School of Medicine, Aydın, Turkey
}

*Corresponding author: Mehmet Turgut, Associate Professor of Neurosurgery, Department of Neurosurgery, Adnan Menderes University School of Medicine, 09100 Aydın, Turkey, Tel: +90 256 2120146; Fax: +90 256 2134874; E-mail: drmturgut@yahoo.com

Received date: Nov 14, 2016; Accepted date: Nov 23, 2016; Published date: Nov 30, 2016

Copyright: (c) 2016 Çavuşoğlu T, et al. This is an open-access article distributed under the terms of the Creative Commons Attribution License, which permits unrestricted use, distribution, and reproduction in any medium, provided the original author and source are credited.

\section{Introduction}

Numerous cerebrum tumors are connected with impressive morbidity and poor prognosis. Morphologically undefined, glioblastoma multiforme of various age gatherings and tumor area involve a few distinctive biologic substances with characterized driver mutations, for example, Isocitrate dehydrogenase, H3F3A and TERT (Telomerase Reverse Transcriptase) [1]. Naturally visible complete neurosurgical resection of these tumors is just conceivable in a few patients in view of the exceedingly invading development design. An imposing intricacy of cerebrum metastases is the spread of tumor cells along the cerebrospinal liquid and is seen in up to $15 \%$ of the cases [2]. Normal anatomic areas of primary tumors are bosom, lung growth, and skin. Prognostic components incorporate tumor area, the number of metastases, reaction to steroid treatment and in breast cancer a positive HER2 status with proper treatment [3].

Laminin and collagen, the main segments of the Extracellular Matrix (ECM) and strong stimulators of glioma development, are generally not watched straight forwardly neighboring the glial tumor cells, aside from in desmoplastic changed tumor zones [4]. To rupture the trust worthiness of the ECM, glial tumor cells emit lattice debasing proteases, serine proteases and grid metalloproteinases, every one of them upregulated in tumors contrasted with a typical mind. Cell bond is then intervened by ECM-receptor families, for example, cadherins that incorporate NCAM and CD44, selectins and the integrins [5]. Cerebrum metastasis incorporates a few stages, including separation of tumor cells from an essential site, intravasation into vessels, extravasation, hibernation a vascular specialties, multiplication and attack to their objective. Not just does the blood cerebrum hindrance limit infiltration of dynamic substances or immunomodulatory cells, additionally astrocytes, pericytes, and microglia contribute straightforwardly to tumor cell colonization in the mind [6]. The tumor cells themselves access the encompassing parenchyma and advance their development through the instigation of angiogenesis. The ECM association is significant for this last difficult stride and it is felt that less than $1 \%$ of the circling tumor cells are effective in framing metastasis. Particular tumor-ECM cooperation gives a decent clarification to the inclination of particular tumor sorts in chose organs.

The ECM is crucial for building backing of tissue, control of cell expansion and separation. The association of the av-group of integrins is intervened by official to specific arginine-glycine-aspartic corrosive arrangements of the ECM ligands, while $\alpha 4 \beta 1, \alpha 4 \beta 7$ and $\alpha 9 \beta 1$ tie to an acidic theme, termed "LDV," that is practically identified with RGD [7]. Some integrins, particularly $\alpha v \beta 6$ and $\alpha v \beta 8$ have been connected with nearby actuation of TGF- $\beta$ and this integrin-intervened activation of TGF- $\beta 1$ by means of the aryl-hydrocarbon receptor is important for legitimate capacity of this key controlling particle. In the creating cerebrum, integrins spatio transiently contribute in a cell-particular way for unmistakable examples of neuronal relocation, cortical layer development and separation inside the cortex through association with neurotrophic elements. Neural peak cells, the antecedent cells got from the dorsal neural tube, express numerous integrins and particularly 1 is by all accounts vital for Schwann cell separation. $\beta 1, \beta 5, \beta 8$ integrins are communicated in all areas of the creating cerebrum and their look holds on in the grown-up creature cortex, while $\beta 6$ is limited to neuronal cells and $\beta 2, \beta 3, \beta 4$ are totally truant. Integrins are communicated at most neurotransmitters in the mind, and hereditary and pharmacological studies demonstrate that they are required for ordinary synaptic pliancy yet are not included in neurotransmitter development. In spite of the fact that $\alpha v \beta 8$ and $\beta 1$ integrins are available in ordinary human oligodendrocytes, information from creature models lacking $\beta 8$ or $\beta 1$ demonstrate that they are not required for typical myelinisation $[8,9]$.

Alterations in integrin expression accompany and may contribute to the ability of cancer cells to cross physiological barriers in their tissue of origin and allow them to invade other structures. Integrin av $\beta 6$ is associated with cells of epithelial lineage and is rapidly upregulated on during tissue injury for activation of latent transforming growth factor 1. In gliomas, especially $\alpha 5 \beta 1, \alpha v \beta 3$ and $\alpha v \beta 5$ integrins, which are frequently expressed in tumor endothelia and in some tumor cells, may affect glioma tumor initiation and progression [10]. Except for a single pilocytic astrocytoma immunreactive for $\beta 1$ integrin, no studies in pediatric glial/glioneuronal tumors have been performed so far. Integrin $\beta 3$-knockout mice display enhanced tumor growth and a proangiogenic phenotype which has led to the concept that $\beta 3$ expression may mediate a balance between protumor and antitumor effects. This data indicates that $\alpha v \beta 3$ integrin plays a key role in malignant gliomas and is a promising target for treatment. Expression patterns of the av integrin complexes differ between primary and metastatic lesions.

The proclivity of RGD peptides (Arg-Gly-Asp) for their ligands is profoundly reliant on peptide adaptation and flanking amino corrosive groupings that impact receptor selectivity. Cilengitide is the most generally contemplated RGD $\alpha v \beta 3$ and $\alpha v \beta 5$ rival and has experienced stage III clinical trials [11]. Moreover, these studies may advance highlight the need for patient determination in future trials with integrin restraint strategies, e.g. by RDG PET. Other integrin-focusing on methodologies incorporate RGD-united nanoparticles or liposomes that are disguised by integrin-intervened endocytosis and afterward 
Citation: Çavusoglu T, Uyanikgil Y, Kiliç KD, Turgut M (2016) Integrins as a Critical Target in Determining Metastasis and Growth of Brain Tumor. J Brain Tumors Neurooncol 1: e105. doi:10.4172/2475-3203.1000e105

Page 2 of 2

limited in perinuclear areas. Preclinical studies included RGD bearers combined with paclitaxel, doxorubicin and gemcitabine in carcinoma and glioma cell lines. These RGD techniques are additionally abused to convey radionucleotides or serve as a radiotracer, for example, $[18 \mathrm{~F}]$ Galacto-RGD in PET and SPECT [12]. Another RGD radiolabeled marker with high partiality for $\alpha v \beta 3 / \alpha v \beta 5$ integrin, Fluciclatide is right now being tried to survey angiogenesis in strong tumors. Elective methodologies incorporate monoclonal antibodies and non-RGD antibodies. Vedolizumab ties to the a $4 \beta 7$ integrin complex and is utilized for the treatment of ulcerative colitis yet has right now no part in malignancy treatment [13].

In conclusion, integrins are critical components in determining the metastasis and growth of tumors. Further studies are needed to explore anti-integrin strategies how can be used in cancer treatment.

\section{References}

1. Appin CL, Brat DJ (2015) Biomarker-driven diagnosis of diffuse gliomas. Mol Aspects Med 45: 87-96.

2. Chamberlain MC (2012) Neoplastic meningitis and metastatic epidural spinal cord compression. Hematol Oncol Clin North Am 26: 917-931.

3. Bartsch R, Berghoff A, Pluschnig U, Bago-Horvath Z, Dubsky P, et al. (2012) Impact of anti-HER2 therapy on overall survival in HER2overexpressing breast cancer patients with brain metastases. $\mathrm{Br}$ J Cancer 106: $25-31$.
4. Giese A, Loo MA, Rief MD, Tran N, Berens ME (1995) Substrates for astrocytoma invasion. Neurosurgery 37: 294-301.

5. Denda S, Reichardt LF (2007) Studies on integrins in the nervous system. Methods Enzymol 426: 203-221.

6. Fidler IJ (2011) The role of the organ microenvironment in brain metastasis. Semin Cancer Biol 21: 107-112.

7. Hynes RO (2002) Integrins: bidirectional, allosteric signaling machines. Cell 110: 673-687.

8. Cousin B, Leloup C, Pénicaud L, Price J (1997) Developmental changes in integrin beta-subunits in rat cerebral cortex. Neurosci Lett 234: 161-165.

9. Schittenhelm J, Klein A, Tatagiba MS, Meyermann R, Fend F, et al. (2013) Comparing the expression of integrins avß3, avß5, avß6, avß8, fibronectin and fibrinogen in human brain metastases and their corresponding primary tumors. Int J Clin Exp Pathol 6: 2719-2732.

10. Avraamides CJ, Garmy-Susini B, Varner JA (2008) Integrins in angiogenesis and lymphangiogenesis. Nat Rev Cancer 8: 604-617.

11. Carter A (2010) Integrins as target: first phase III trial launches, but questions remain. J Natl Cancer Inst 102: 675-677.

12. Haubner R, Maschauer S, Prante O (2014) PET radiopharmaceuticals for imaging integrin expression: tracers in clinical studies and recent developments. Biomed Res Int 2014: 871609.

13. Abdollahi A, Griggs DW, Zieher H, Roth A, Lipson KE, et al. (2005) Inhibition of avß3 integrin survival signaling enhances antiangiogenic and antitumor effects of radiotherapy. Clin Cancer Res 11: 6270-6279. 